Plant cell cultures for the production of health promoting metabolites

Oren-Shamir, M.I. Agricultural Research Organization

Sinclair, D.A. Harvard Medical School

Fait, A. Ben-Gurion University of the Negev

Galili, G. Weizmann Institute of Science

Or, E. Agricultural Research Organization

Project award year: 2015

Three year research project 


\section{Plant cell cultures for the production of health promoting metabolites - Abstract}

List of original objectives: 1. Characterize the effect of enhanced AAA production in grape berry cell culture on the three major phenylpropanoid-derived pathways. 2. Enhance production of stilbenes and flavonoids by metabolic engineering of the cell culture. 3. Characterize the effect of external stresses induced on the double-transformed grape cell cultures on their metabolomics and transcriptomic profiles. 4. Test the potential of cell extracts on correlates of human health and longevity. 5. Transform grape embryogenic calli.

Background: Polyphenolic secondary metabolites that accumulate in fruit promote health, and have been shown to prevent development of chronic diseases such as heart disease, cancers, type 2 diabetes and obesity. Grapes (Vitis vinifera) are important sources of phyto-antioxidants, pigments, and essential nutrients worldwide. There is incredible diversity in chemical composition within the various genotypes of grapes, a significant part of which is derived from the aromatic amino acids (AAAs). While phenolic secondary metabolites such as resveratrol, quercetin and anthocyanins are beneficial to human health, their enhancement in plants by traditional breeding has proven difficult.

Transformable cell cultures of grape berries offer defined production systems, with rapid yield and relatively uniform quality, which are free from geographical, environmental and seasonal constrains. The IL group specialized in transforming red grape cell cultures, resulting in overproduction of phenylalanine and its derived phenolic metabolites. The US group has extensive expertise in studying the effects of such compounds on human health promotion and longevity. We hypothesize that extracts from grape cultures with increased polyphenolic metabolism will improve stress resistance in mammalian cells, with significant health benefits.

Achievements, conclusions and implications: Transformation of grape cell suspensions is a complex process that only few groups have successfully performed. Here we improved the transformation process of transforming Vitis vinifera cv.Gamay Red cell suspension and have successfully produced over 50 stable transgenic grape cell lines via Agrobacterium-mediated genetic transformation. The cell suspension was transformed with: 1 . AroG*, a bacterial feedback insensitive gene encoding for DAHPS, for increased production of AAAs and in particular Phe, 2. AroG* + STS (Stilbene Synthase) for directing the carbon flower towards overproduction of stelbenes and 3. AroG* + FLS (Flavonol Synthease) for overproduction of flavonoids.

The effect of overexpressing the three different constructs on the metabolic profile of the grape cell cultures, revealed that the concentration of stilbenes and in particular viniferin increased significantly in all transgenic lines, with the highest increase in the double transgenics of AroG $^{*}+$ STS. The fold change increase in stilbenes is the highest published to date, suggesting that the increased production of AAAs due to AroG* contributed to this effect. Other studies in which only STS was overexpressed resulted in much lower fold change increases. An interesting point is that even in the AroG*+FLS transgenic line stilbene concentrations were significantly high, with no distinctive effect on the flavonols and anthocyanins. The slight increase in some flavonols and anthocyanins was similar to lines transformed with AroG* ${ }^{*}+$ STS.

Polyphenols from transformed grape cell suspension extracts were added to mammalian cell cultures to test their short term effect on mitochondrial and redox metabolism of these cells. Cell extracts from AroG* transformed grape cells and double transformed cells were sent to the US group for these testings. Unfortunately, the extracts of the transformed lines had a toxic effect on the mammalian cells in all experiments performed.

The main results of this research are 1. Development of an efficient transformation system for the grape cell suspensions. 2. Development of an array of transgenic lines with increased metabolomics production of health promoting metabolites. 3. Production of a system for future studies to understand the interplay between the stilbene and flavonoid pathways. A manuscript describing the metabolomics effects of transformation of Vitis vinifera cv. Gamay Red cell suspension with AroG $^{*}$, AroG $^{*}+$ STS and AroG*+FLS is in advanced preparation. 


\section{Collaboration:}

The IL group sent extracts of grape cell cultures transformed with AroG* for increased production of phenylalanine during the second year of research. The US group calibrated the extraction of the phenolic compounds from the cell extracts. During the second year the US group have shown that the grape cell cultures do improve markers of cellular health of mammalian cells, but did not see a significant difference between the controls and AroG* cell extracts. After completing the formation and initial metabolic characterization of the large number of double transformed grape cell lines, several lines with the highest increase in stilbenes and flavonols were sent during the third year from IL to the US group for further studies. Unfortunately, the extracts of the transformed lines had a toxic effect on the mammalian cells in all experiments performed. During the research years, the two groups communicated and transferred relevant information via email, skype conversations and one travel of the IL PI to the US. 


\section{Achievements}

\section{Transformation}

Transformation of grape cell suspensions is a complex process that only few groups have successfully performed. Here we improved the transformation process of transforming Vitis vinifera cv.Gamay Red cell suspension and have successfully produced over 50 stable transgenic grape cell lines via Agrobacterium-mediated genetic transformation. Our goal in transformation of the Gamay Red cell suspension was to increase production of health promoting phenylpropanoids, namely stilbenes and flavonols (Fig. 1).

The cell suspension was transformed with: 1 . AroG*, a bacterial feedback insensitive gene encoding for DAHPS, for increased production of AAAs and in particular Phe, 2. AroG* + STS (Among $48 V v S T S$ genes in grape, VvSTS5, VvSTS10 and VvSTS28 were selected and cloned, based on the functional assay results in N. benthamiana leaves) and 3. AroG* + FLS. The 5 constructs were created, all expressed under the $35 \mathrm{~S}$ promotor (Fig. 1,2).

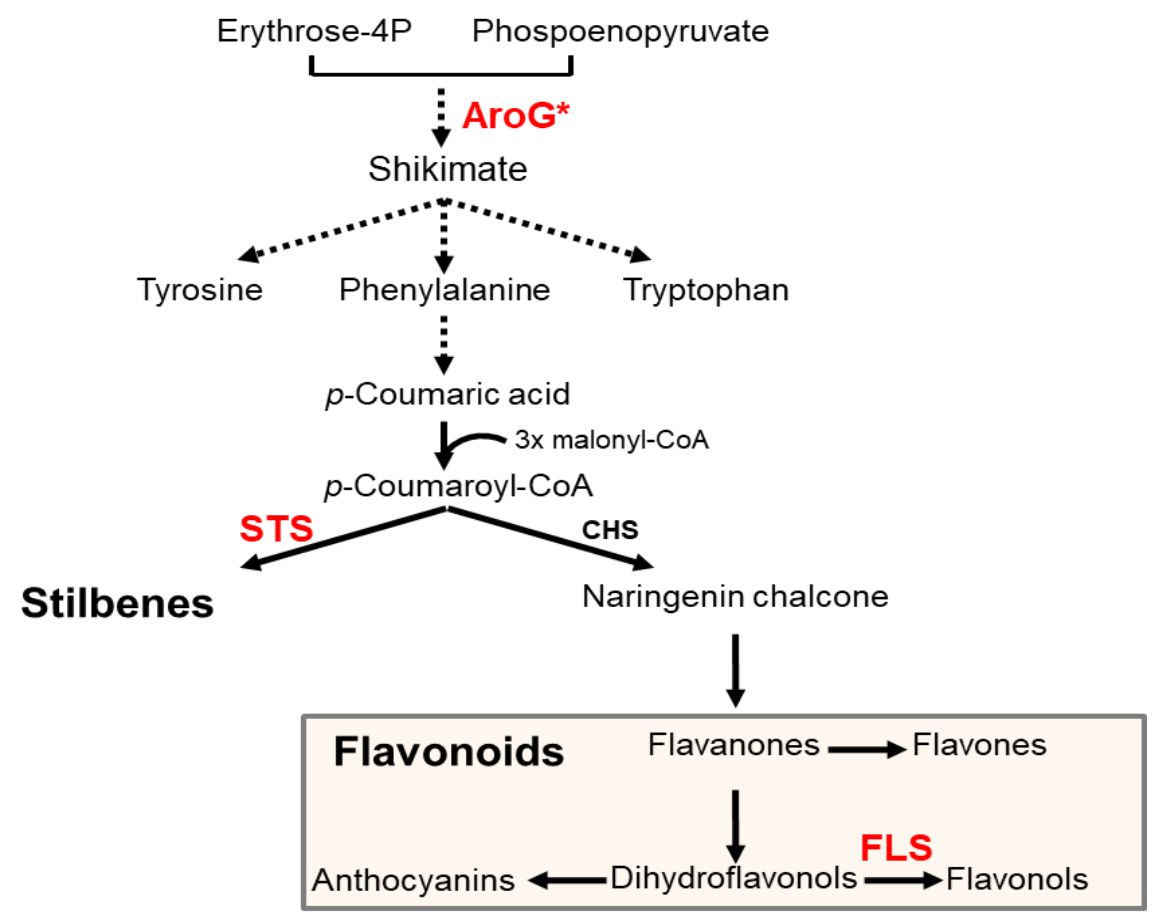

Fig. 1Proposed flavonoid and stilbene biosynthetic pathways designed by co-expression of AroG with FLS or STS in Vitis Vinifera cv. Gamay Red cell culture. The upstream transgene AroG would be capable of increasing rate-limiting metabolite Phe, which could provide enough precursors for branch pathways. AroG, a feedback-insensitive E. coli 3deoxy-D-arabino-heptulosonate 7-phosphate synthase; STS, stilbene synthase; FLS, flavonol synthase. 


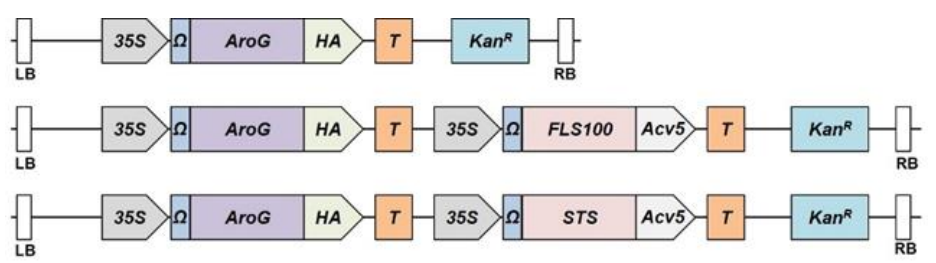

Fig. 2 Schematic illustrations of transgenes expressed in pART27 vector. In gene constructs, transgene expression was under the control of 35S promoter. 35S, CaMV 35S promoter; $\Omega$, TMV; HA: HA-tag sequence; AcV5, AcV5-tag sequence; T, OCS-terminator; $\mathrm{Kan}^{\mathrm{R}}$, Kanamycin resistance marker; LB, T-DNA left border; RB, T-DNA right border; $A r o G^{*}$, a feedback-insensitive bacterial form of 3-deoxy-D-arabino-heptulosonate 7phosphate synthase enzyme (DAHPS); STS, stilbene synthase; FLS, flavonol synthase.

\section{$\underline{\text { Metabolic analysis of transgenic cell culture lines }}$}

To obtain a global view of the effect of expression of the AroG, FLS, STS transgenes on grape stilbene and flavonoid metabolism, we performed a UPLC-MS analysis using independent transgenic lines of AroG* and double transgenic lines, AroG* + FLS and three double transgenic AroG* + STS lines. A principle component analysis plot of phenylpropanoid metabolites detected was performed using : (1) all the annotated metabolites in control, AroG* and AroG* + FLS transgenic lines (Figure 3a); (2) all the annotated metabolites in control, AroG* and $\mathrm{AroG}^{*}+$ STS transgenic lines (Figure $3 \mathrm{~b}$ ). These PCA analyses show a clear metabolomics distinction between controls and each of the transgenic groups (AroG*, AroG*+STS and AroG*+FLS) (see details in Appendix). Three metabolites, Phe, Trp and p-coumaric acid, increased significantly in all three transgenic groups, suggesting that increased production of AAAs due to AroG* overexpression is sufficient for increased levels of these metabolites.

A schematic representation of the metabolic changes between the different transgenic lines is presented in Fig. 3. This scheme is a representative of two complete sets of samples that were taken and analyzed several months apart. The most significant finding is the concentration of stilbenes and in particular viniferin increased significantly in all transgenic lines, with the highest increase in the double transgenics of AroG*+STS. The fold change increase in stilbenes is the highest published to date, suggesting that the increased production of AAAs due to AroG* contributed to this effect. Other studies in which only STS was overexpressed resulted in much lower fold change increases. An interesting point is that even in the $\mathrm{AroG}^{*}+\mathrm{FLS}$ transgenic line stilbene concentrations were significantly high, with no distinctive effect on the flavonols and anthocyanins. The slight increase in some flavonols and anthocyanins was similar to lines transformed with AroG* + STS. 


\section{Effect of transformed grape extracts on mammalian health characteristics}

Polyphenols from transformed grape cell suspension extracts were added to mammalian cell cultures to test their short term effect on mitochondrial and redox metabolism of these cells. Cell extracts from AroG* transformed grape cells and double transformed cells were sent to the US group for these testings. Unfortunately, the extracts of the transformed lines had a toxic effect on the mammalian cells in all experiments performed. A detailed description of the experiments with mammalian cells is attached in the appendix.

\section{$\underline{\text { Main achievements }}$}

1. Development of an efficient transformation system for the grape cell suspensions.

2. Development of an array of transgenic lines with increased metabolomics production of health promoting metabolites.

3. Production of a system for future studies to understand the interplay between the stilbene and flavonoid pathways.

A manuscript describing the metabolomics effects of transformation of Vitis vinifera cv. Gamay Red cell suspension with AroG*, AroG*+STS and AroG*+FLS is in advanced preparation, and the additional findings are described in the appendix.

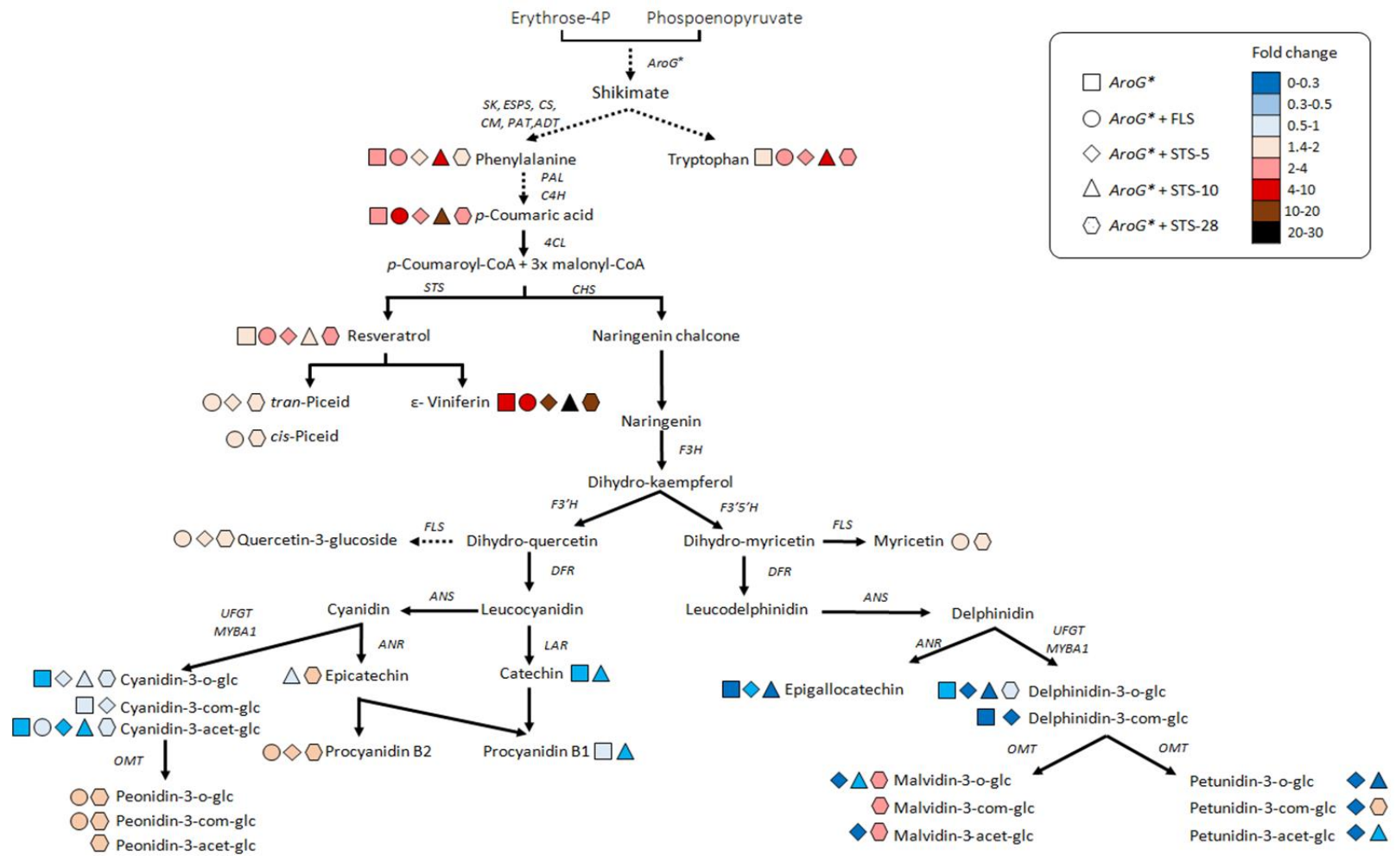

Fig. 3 Schematic representation of metabolic changes with fold change normalized to control (empty vector). The levels presented are the median value in each metabolite among different transgenic lines (3 empty lines, 3 AroG* $^{*}$ lines, 5 AroG*+FLS lines, 3 of each of the AroG*+STSs lines) . Different transgenic lines were marked as squares (single AroG* line), round (AroG*+FLS), diamond (AroG*+STS5), triangle (AroG*+STS10), hexagon (AroG*+STS28). 
Publications for Project IS-4850-15 R

\begin{tabular}{|cccccc|}
\hline $\begin{array}{c}\text { Stat } \\
\text { us }\end{array}$ & Type & Authors & Title & Journal & $\begin{array}{c}\text { Vol:pg } \\
\text { Year }\end{array}$ \\
\hline
\end{tabular}




\section{Appendix}

\section{Table of content}

Additional data on production and metabolomics analysis of grape cell suspension.....1-3

Testing grape cell extracts effect on mammalian cell cultures

Additional data on production and metabolomics analysis of grape cell suspension

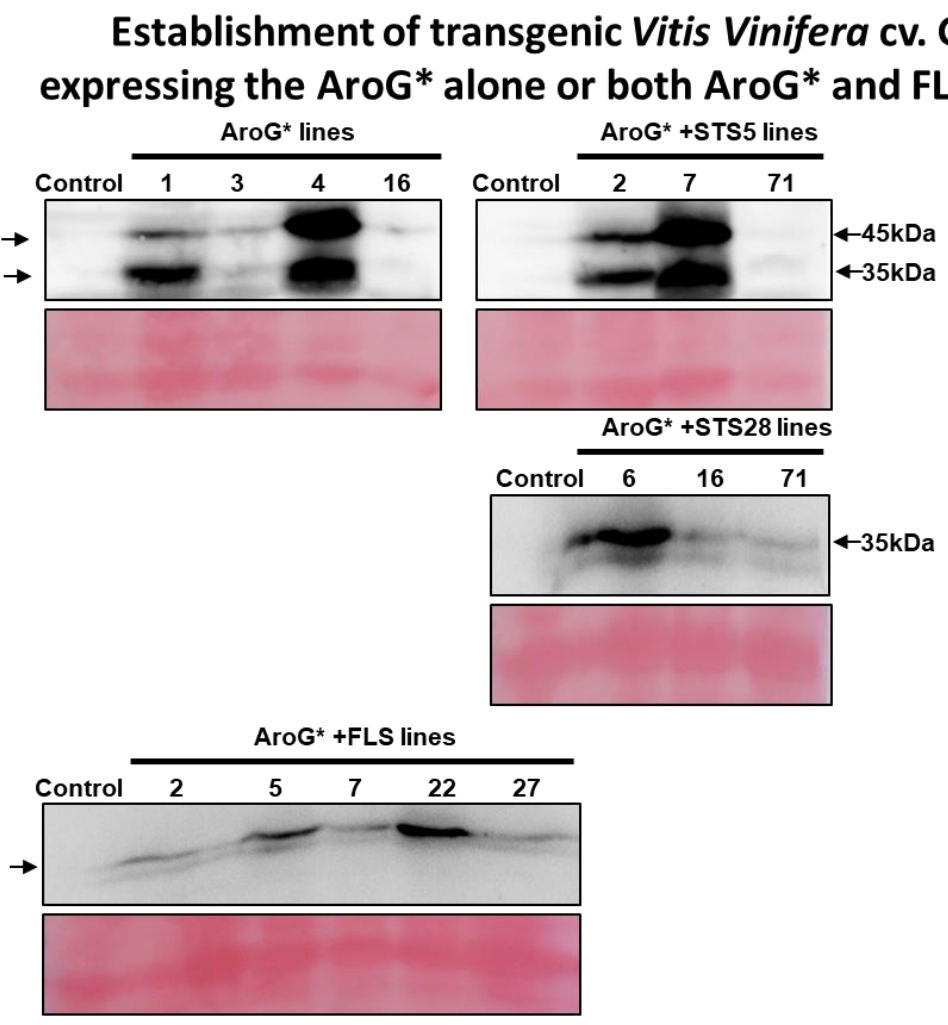

Figure 1. Transgenic Vitis vinifera cv. Gamay cell culture expressing AroG* (a1-a5) and either STS (b1-b3) or FLS (b4) were generated. The expression of AroG* and either FLS or STS was monitored by immunoblot analysis with anti-Flag antibody. Immunoblot analysis was performed on protein extracts (50 ug) of each line, using anti-HA antibody (1:500) for AroG* analysis, anti-AcV5 (1:xx00) for FLS or STS analysis. The major protein band of AroG* at 35kDa. The major protein band of FLS or STS at $35 \mathrm{kDa}$. Protein Ponceau staining for each gel indicates similar protein loading. 


\section{Discrimination of metabolic profile in empty, AroG* and double transgenic lines}
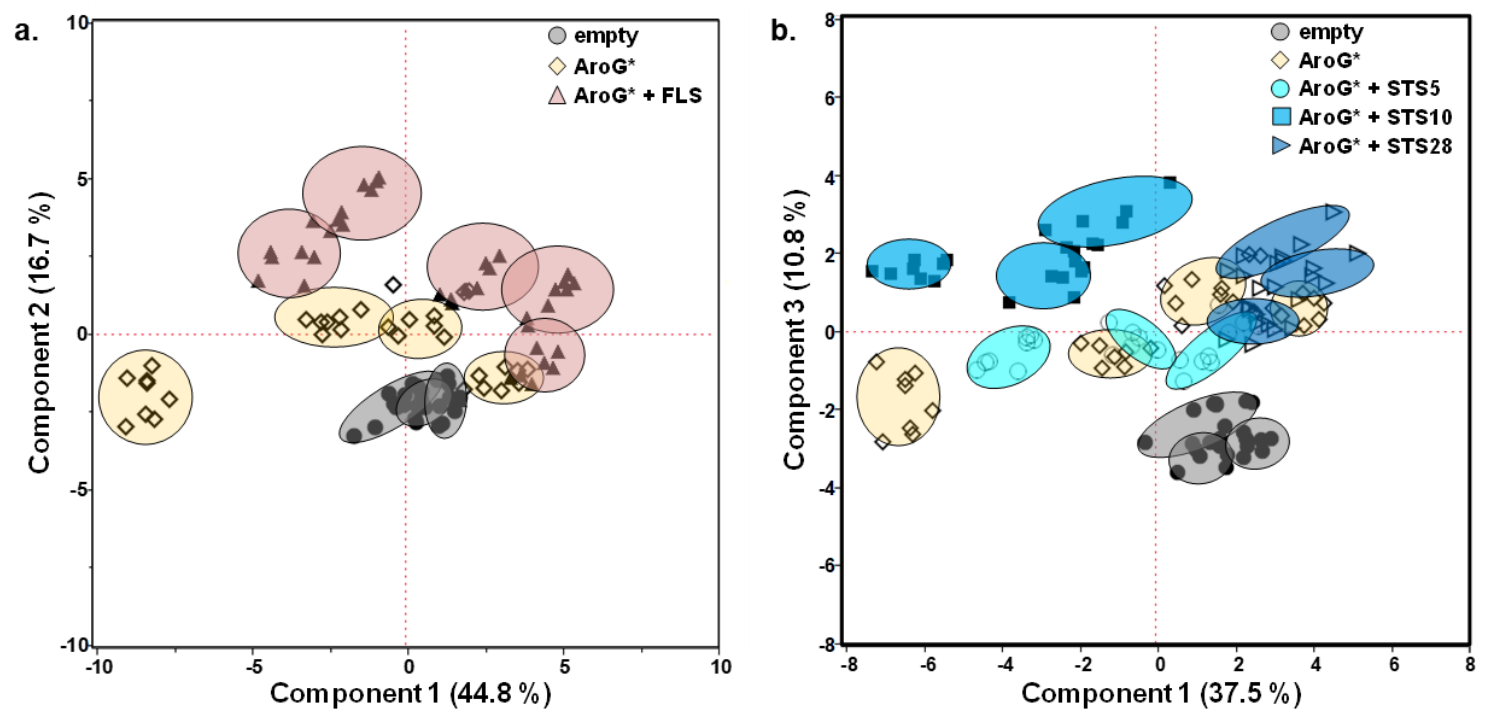

Figure 2. Principle component analysis plot of phenylpropanoid metabolites detected in (a) empty, AroG*, AroG*+FLS samples; and in (b) empty, AroG* and AroG*+STS samples. Values used for this analysis are normalized peak heights and then log-transformed data. Each cloud represent 8 replications of each line.
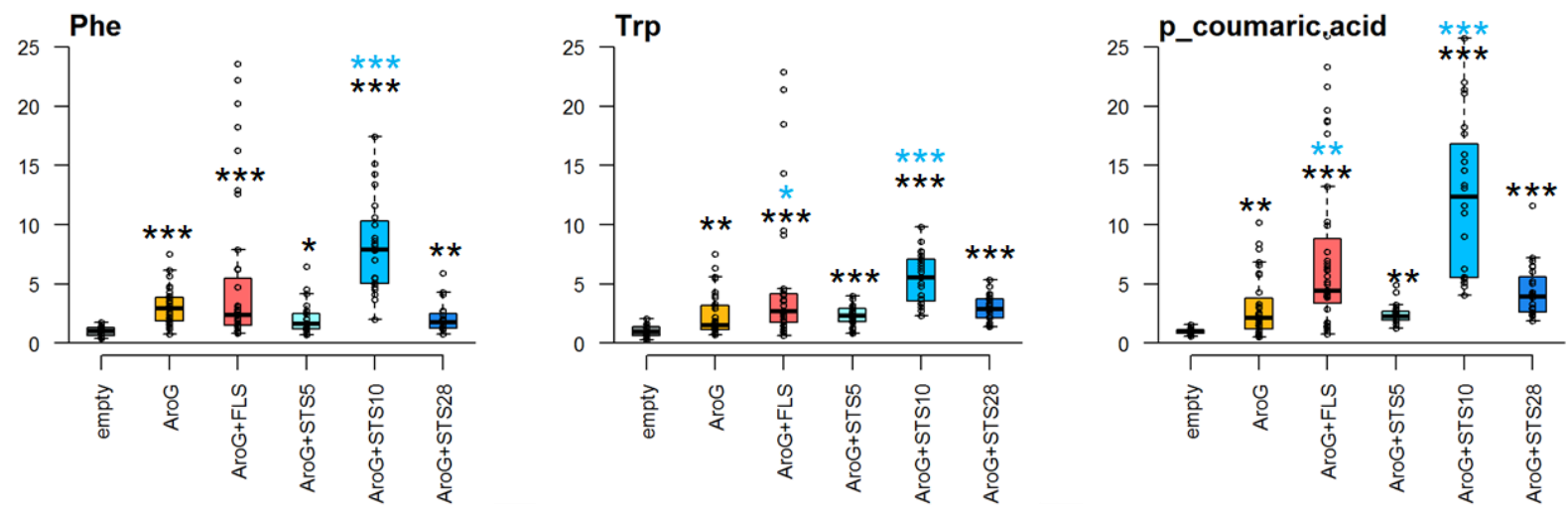

Figure 3. Levels of Phe, Trp, and coumaric acid in AroG*, AroG*+FLS, AroG*+STS transgenic lines. Levels of metabolites are presented as fold change of transgenic lines in comparison with the control line (empty vector). Each Boxplot was a mixture of the metabolite content ( $y$-axis of three to five individual transformation colonies) with each replication. There were eight pseudo-biological replications for each line. Statistical significance was analysed by Kruskal-Wallis test, followed by Dunnett methods for joint ranking test. Black asterisk represent significant difference in metabolites content between control (empty) and transgenic lines, blue asterisk represent significant difference in metabolites content between single AroG* lines and double transgenic lines ( $p \leq 0.05 ; p \leq 0.01 ; p \leq 0.001)$. 


\section{Coexpression of AroG and FLS/STS promoted the higher accumulation of stilbenes}
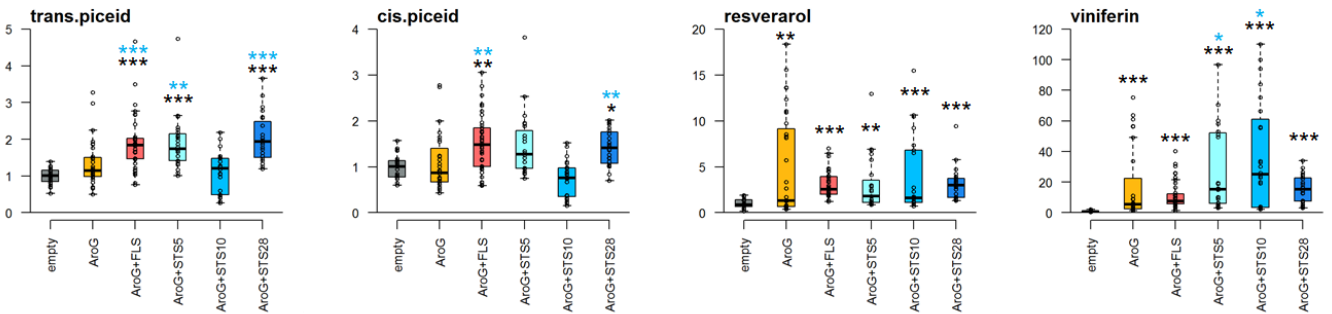

Figure 4. Effect of co-expression of both AroG and FLS or both AroG and STS on the stilbenes accumulation in Vitis transformated cells. Boxplot was a mixture of the metabolite content ( $y$-axis of three to five individual transformation colonies) with each replication. There were eight pseudobiological replications for each line. Statistical significance was analysed by Kruskal-Wallis test, followed by Dunnett methods for joint ranking test. Black asterisk represent significant difference in metabolites content between control (empty) and transgenic lines, blue asterisk represent significant difference in metabolites content between single AroG* lines and double transgenic lines ( $p \leq 0.05 ; p \leq 0.01$; $\mathrm{p} \leq 0.001$ ). Here, the content of resvertrol in control lines is $0.05 \mathrm{mg} / \mathrm{g}$ (dry weight)

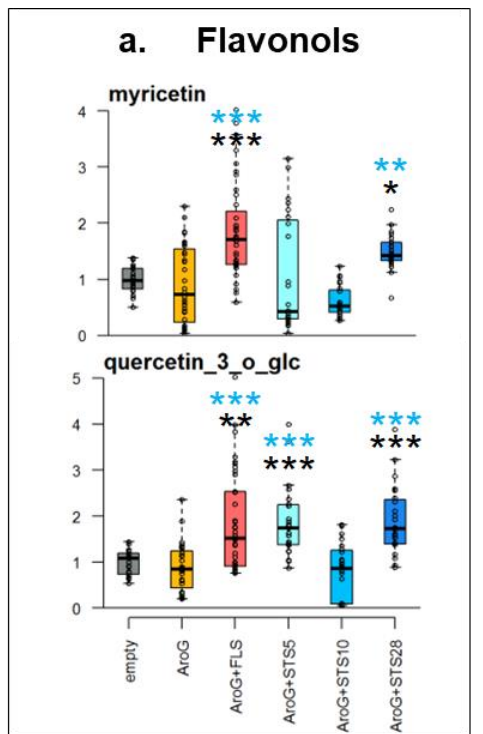

Figure 5. Effect of co-expression of both AroG and FLS or both AroG and STS on the flavonoids accumulation in Vitis transformated cells. Boxplot was a mixture of the metabolite content (y-axis of three to five individual transformation colonies) with each replication. There were eight pseudobiological replications for each line. Statistical significance was analysed by Kruskal-Wallis test, followed by Dunnett methods for joint ranking test. Black asterisk represent significant difference in metabolites content between control (empty) and transgenic lines, blue asterisk represent significant difference in metabolites content between single AroG* lines and double transgenic lines ( $p \leq 0.05$; $\mathrm{p} \leq 0.01 ; \mathrm{p} \leq 0.001)$.

\section{b. Flavan-3-ols}
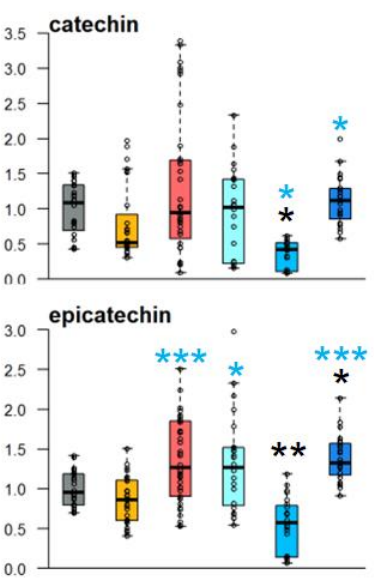

epigallocatechin

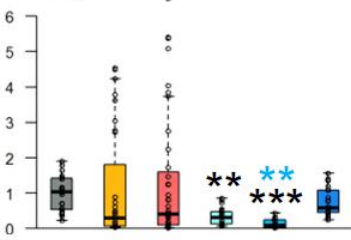

procyanidin_b1
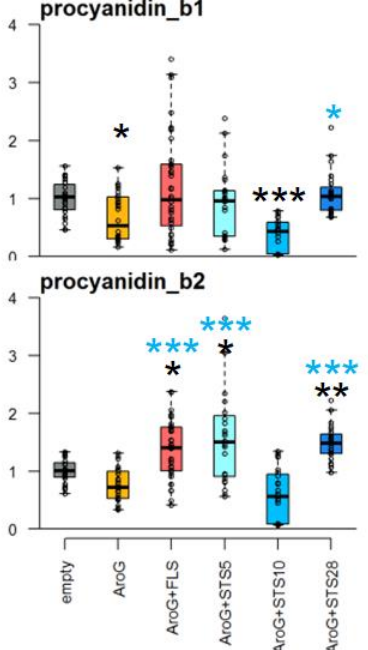

c. Anthocyanins
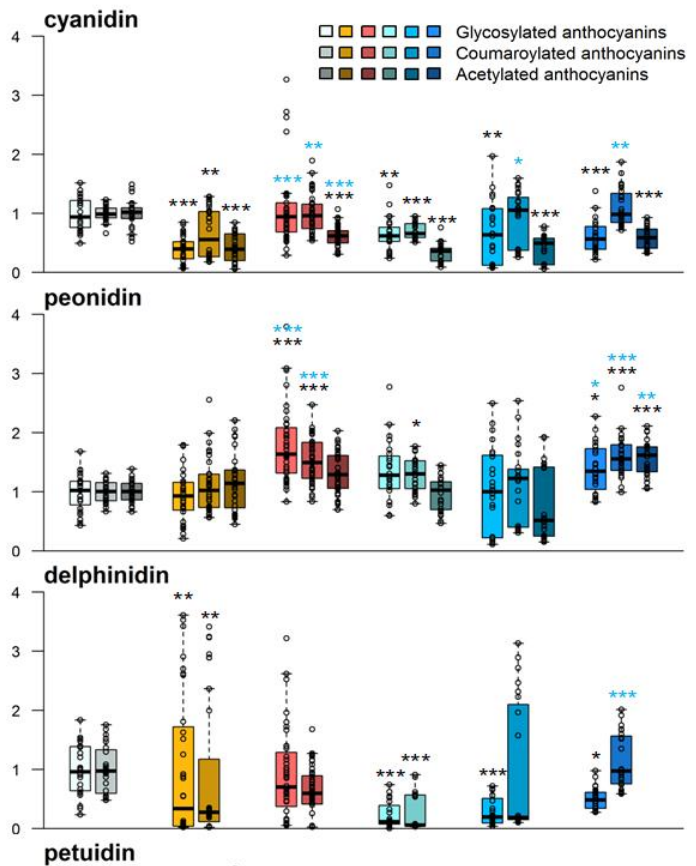

petuidin
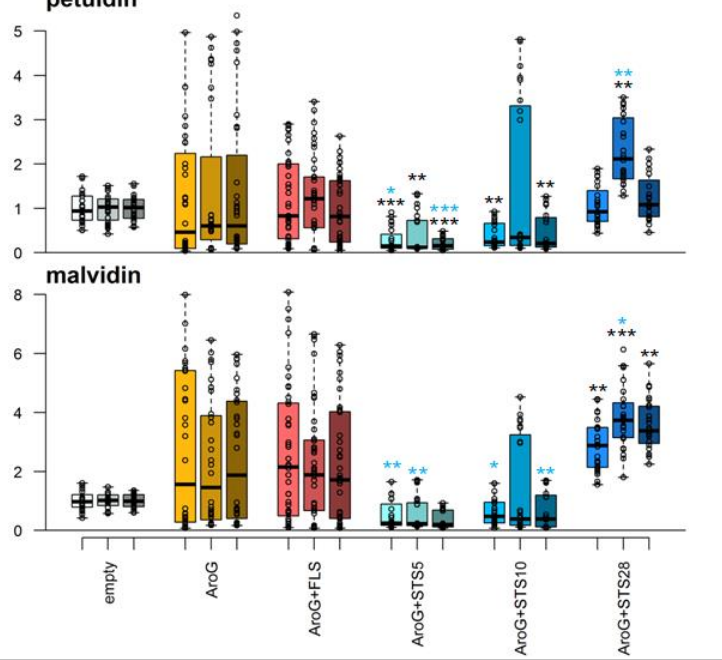
$\underline{\text { Testing grape cell extracts effect on mammalian cell cultures }}$

\section{Testing grape cell extracts effect on mitochondrial and redox metabolism of mammalian cell cultures}

This report summarizes the results of a series of experiments testing grape cell extracts (GCEs), kindly provided by the Oren-Shamir lab, to determine the effect of short-term incubation on the mitochondrial and redox metabolism of mammalian cells in culture. The assays used in this project aimed to measure the effect of polyphenols (PPs) contained in these extracts, like anthocyanins, flavanols, flavonols, stilbenes and phenolic acids. These variables have been previously associated with improved aspects of the cellular metabolism upon incubation when these polyphenolic compounds are tested, generally as individual compounds ${ }^{1-3}$ and more rarely, as full extracts ${ }^{4^{-10}}$. Nonetheless we aimed to measure similar variables to look for improvement of cellular health. See Table 1.

\begin{tabular}{|llll|}
\hline Variable measured & Active reference & Output & Platform \\
\hline Mitochondrial mass & MitoTracker DeepRed, NAO & Fluorescense & Plate reader \\
\hline Mitochondrial Membrane Potential & TMRM & Fluorescense & Plate reader \\
\hline Oxygen consumption & $\mathrm{O}_{2}$ florescent sensor & Fluorescense & Seahorse \\
\hline Cellular viability & $\begin{array}{l}\text { CalceinAM/Ethidium } \\
\text { homodimer-1 }\end{array}$ & Fluorescense & Plate reader \\
\hline Redox state & CellRox Green & Fluorescense & Plate reader \\
\hline
\end{tabular}

Table 1. Assays used in this project. All assays were performed in 96 -well plate format and the readout was obtained by plate reader. NAO, Nonyl acridine orange; TMRM, Tetramethyl rhodamine; Seahorse, a platform to measure oxygen consumption rate and extracellular acidification rate in cells in real time; Presto blue measures the reducing ability of cells upon the reduction of the compound resazurin into resorufin. CellRox green measures the oxidation of a compound reactive to superoxide anions upon the presence of a potent oxidizer like menadione.

We used as model both human hepatocarcinoma cells (HepG 2 ) and mouse myoblasts ( $\mathrm{C}_{2} \mathrm{C}_{12}$ cells), as these constitute a good cellular model recurrently used in the literature to test the effect of the polyphenols $\frac{11-14}{1-}$.

\section{Grape Cell Extracts}

The extracts tested here correspond to GCEs received on Jan $23^{\text {rd }}, 2018$. The labels used here match the samples names observed in the tubes as follows:

\begin{tabular}{|llll|}
\hline GCE & ID & Dry weight supplied & Mass used for extracts \\
\hline Wild Type & WT & $3.3 \mathrm{~g}$ & $1 \mathrm{~g}$ \\
\hline Wild Type + Phe & WT+Phe & $3.2 \mathrm{~g}$ & $1 \mathrm{~g}$ \\
\hline AroG + FLS & AroG & $1.2 \mathrm{~g}$ & $1 \mathrm{~g}$ \\
\hline
\end{tabular}

Table 2. GCEs tested in this project. WT, untreated grape cells; WT+Phe, grape cells grown under high concentration of the aminoacid phenylalanine; AroG over-expressing grape cells.

From previous work carried out in 2017 with another batch of GCEs where the enzyme phospho-2-dehydro-3-deoxyheptonate aldolase was overexpressed in the lines termed AroG1 to AroG3, we know that these lines are enriched in several polyphenolic compounds including anthocyanins and flavonoids (like resveratrol), among others but the active concentration varied per condition. Importantly, the concentration of polyphenols in these 
extracts was determined with precision. Instead, what is reported here is the biological potency when tested at various concentrations (percent $v / v$ ).

The soluble fraction of these extracts was obtained by ethanol extraction, as described in the report sent on October, 2017. Briefly, we used $1 \mathrm{~g}$ of grape cell dry weight dissolved in $5 \mathrm{~mL}$ of $50 \%$ ethanol. The slurry was mixed overnight at room temperature in a rotational agitator followed by two rounds of centrifugation at $13,000 \times \mathrm{g}$ for $5 \mathrm{~min}$ to pellet the insoluble cell debris. The supernatants were concentrated by evaporation in a speed-vac system at $50^{\circ} \mathrm{C}$ for about $45 \mathrm{~min}$, and the final volume was adjusted with $50 \%$ ethanol to 1 $\mathrm{mL}$ across all samples. The extracts were sterilized by filtration through a $0.22 \mathrm{um}$ polytetrafluoroethylene (PFTE) membrane, aliquoted and stored at $-20^{\circ} \mathrm{C}$ until used.

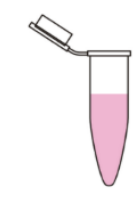

Dry weight

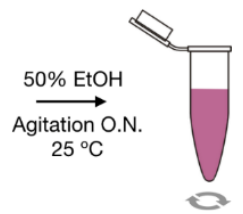

Slurry

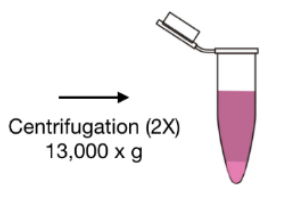

Debris removal

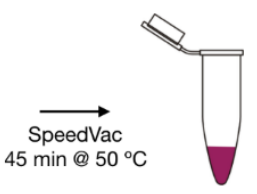

Concentrate

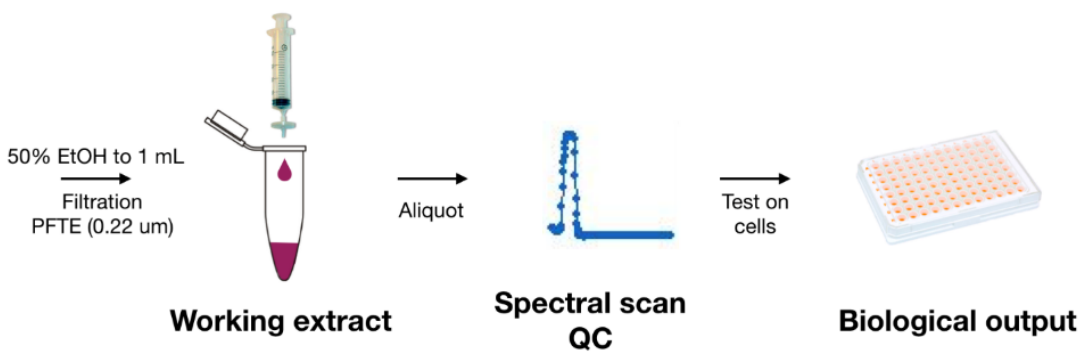

Fig. 1. Pipeline for the obtention of grape cell extract GCEs. See text for details.

\section{Estimation of Resveratrol (RSV) Concentration in the CGEs.}

The only data regarding RSV concentration was provided only for the former AroG lines (provided on April, 2017) through mass spectrometry analyses. To overcome this problem, we tried to estimate its concentration by measuring the absorbance at $305 \mathrm{~nm}$, corresponding to trans-resveratrol, the biologically active isomer ${ }^{15}$. The estimated concentration at $0.63 \%$ was about $14 \mathrm{UM}$; however the lack of linearity observed with this assay made impossible to determine the active concentration of the of the flavonoid in these extracts, possibly due to the interference of absorbance from other metabolites. 
A

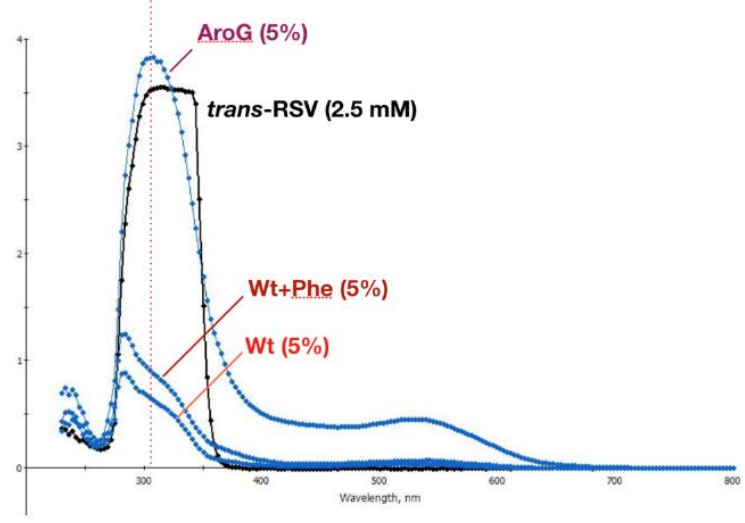

B

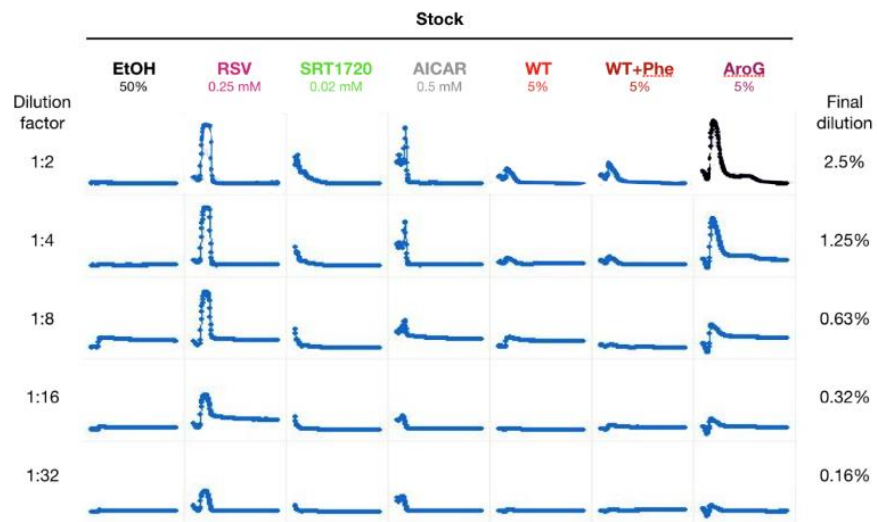

C

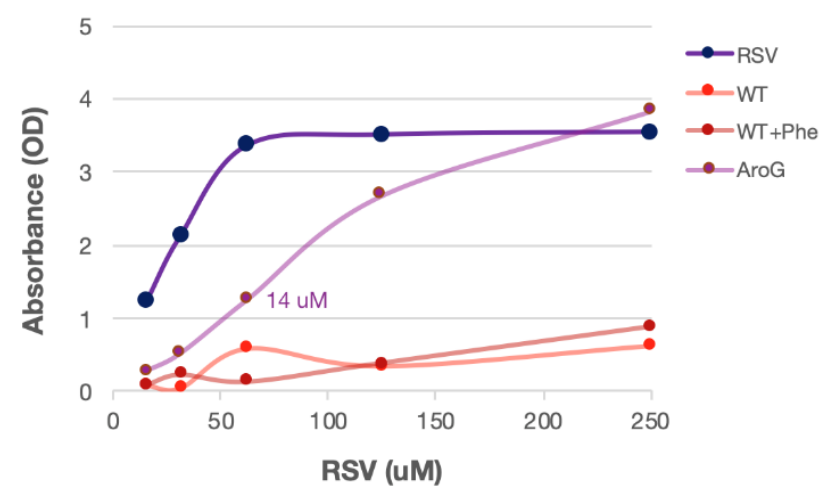

Fig. 2. Determination of RSV concentration by OD in GCEs. A. Spectral scan of 100 UL of GCEs after elution. The dashed red line indicates the optimal absorbance of trans-resveratrol as reference. B. Spectral scan of GCEs at various dilutions (indicated on the right). C. Absorbance of GCEs at $305 \mathrm{~nm}$ at various concentrations as determined in B.

Most studies involving PPs bioactivity have been done both in vitro and in vivo, mostly oriented to study the effect of RSV or other PPs, and more rarely in combination. It is known that resveratrol and other polyphenols may improve mitochondrial function by increasing their intracellular mass and by promoting an increase of the inner membrane potential, accompanied by an increase in oxygen consumption $3,16,17$. Some pathways that are activated upon this include the induction of genes like PGC1a -a transcription factor involved in mitochondrial biogenesis- $\frac{18}{1}$, the phosphorylation of AMPK -a sensor of the ADP/ATP ratioand activation of important regulators of the cellular homeostasis like the deacetylase SIRT1, among other functions.

Despite the fact that these compounds have proven to improve cellular metabolism, reduce inflammation, increase resistance to oxidative stress, reduce insulin sensitivity, lower circulating triglycerides, among others, it has also been documented that at high concentrations these compounds can be toxic as well, especially on cancer cells $s^{16,19-22}$.

Due to the complexity of these mixtures, we were aware that complex polyphenolic mixtures may give may increase apoptosis, slow cell proliferation, decrease mitochondrial activity, decrease free radical abundance, among others ${ }^{3}$.

From our experience with previous extracts, we observed that at high concentrations $>1 \%$ they showed higher toxicity on the cells as they became more prone to detaching from the 
plate surface, particularly at $48 \mathrm{~h}$ of incubation. This was comparable to the effects of resveratrol alone when used at $25 \mathrm{mM}$ (data not shown). Therefore, we decided to do all further assays only at $24 \mathrm{~h}$ of treatment.

Given that these new extracts were resuspended in the same final volume ( $1 \mathrm{~mL}$ e/a), the only way to determine biological potency was to test them at various dilutions on cultured cells. We first tested a range between 0.1 to $5 \%$ and compared their potency with pure RSV as control at $12.5 \mathrm{mM}$ (data not shown). For all subsequent assays, we decided to use $1 \%$ and $5 \%$ GCEs since at these concentrations their effects were more visible.

\section{Cellular Viability}

The effects of the GCEs on cell viability was demonstrated by measuring the esterase activity of cells treated with GCEs after $24 \mathrm{~h}$ of incubation. Our assay was based on the deesterification of Calcein-AM, a polyanionic membrane permeable fluorescein derivative that is de-esterified by intracellular esterases in the cytoplasm and accumulates over time. If the plasma membrane is compromised, as when cells undergo cell death, the fluorescent dye dissipates, and the fluorescence signal does not increase linearly with time. Our results showed that the GCEs decrease viability in average $20 \%$ at both 1 and $5 \%$ after $24 \mathrm{~h}$ of incubation (Figure 3).

A

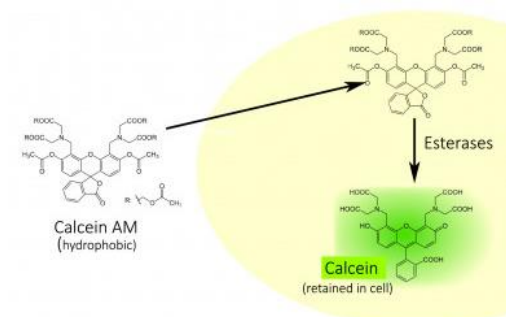

B

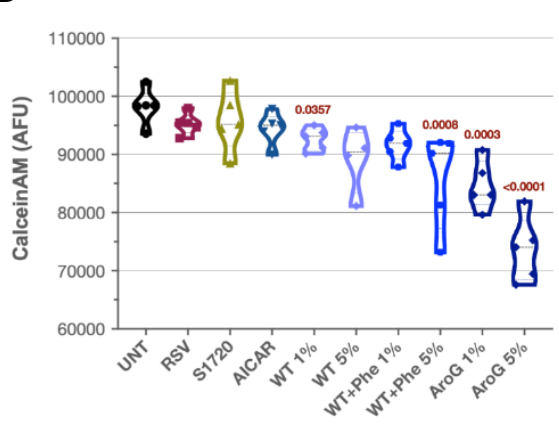

C

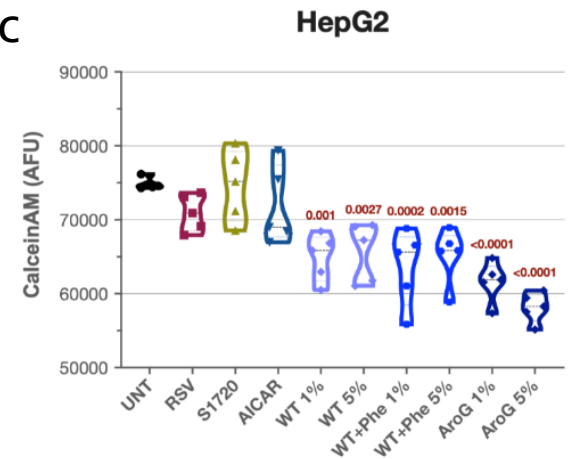

Fig. 3. Effect of GCEs on Cellular Viability. A. The mode of action of Calcein-AM is by passively permeating the cell membrane where intracellular esterases will cleave off a carboxylate residue, allowing the molecule to fluoresce (Ex/Em $494 / 517 \mathrm{~nm}$ ). In this case, the fluorescence linearly correlates with its accumulation in the cell if the plasma membrane is not compromised. B \& C. Raw fluorescence values of cells incubated with Calcein-AM after $24 \mathrm{~h}$ treatment of GCEs onto $C_{2} C_{12}$ myoblasts (B) and HepG2 hepatocytes (C). Violin plots depict the average of 25 fields per well per biological replicate with $95 \% \mathrm{Cl}$ at their ends. P-values ( $\leq 0.05$ ) depicted in red above the data points, were calculated by a one-way ANOVA with Dunnett correction for multiple comparisons using untreated cells (UNT) as control $(n=5-6)$.

In general, both cell lines showed a decreased viability that correlated with the concentration of the GCEs, more especially in cells incubated with the AroG extract at $5 \%$, were the concentration of RSV and other PPs were expected to be higher. Interestingly, this effect is more pronounced in the hepatocarcinoma cell line, corroborating the deleterious effect of GCEs on cancerous cells $\frac{11-13}{}$.

\section{Mitochondrial Mass}

Several lines of evidence have suggested that some of the beneficial aspects of polyphenols in cells is the indirect stimulation of mitochondrial biogenesis, by enhancing the autophagy process required to dispose of faulty mitochondria, particularly those that have a high rate of reactive oxygen species production. 
To measure this, we used to complementary methods. One that relies on cardiolipin staining, a fatty acid enriched in mitochondria, using a fluorescent sensor nonyl acridine orange (NAO). The second, uses the MitoTracker Deep Red (MTDR) dye, a thiol-reactive chloromethyl group that reacts with the cystein residues belonging to mitochondrial proteins in the inner mitochondrial membrane (data not shown). These two dyes are not perfect; NAO is sensitive to the membrane potential of the mitochondria whereas MTDR is not, but its dependent on the area of the inner mitochondrial membrane which has been shown to change under various conditions. However, generally the dyes give a good estimation of the mitochondrial mass when data is taken together. -Mitotracker data needs to be repeated.
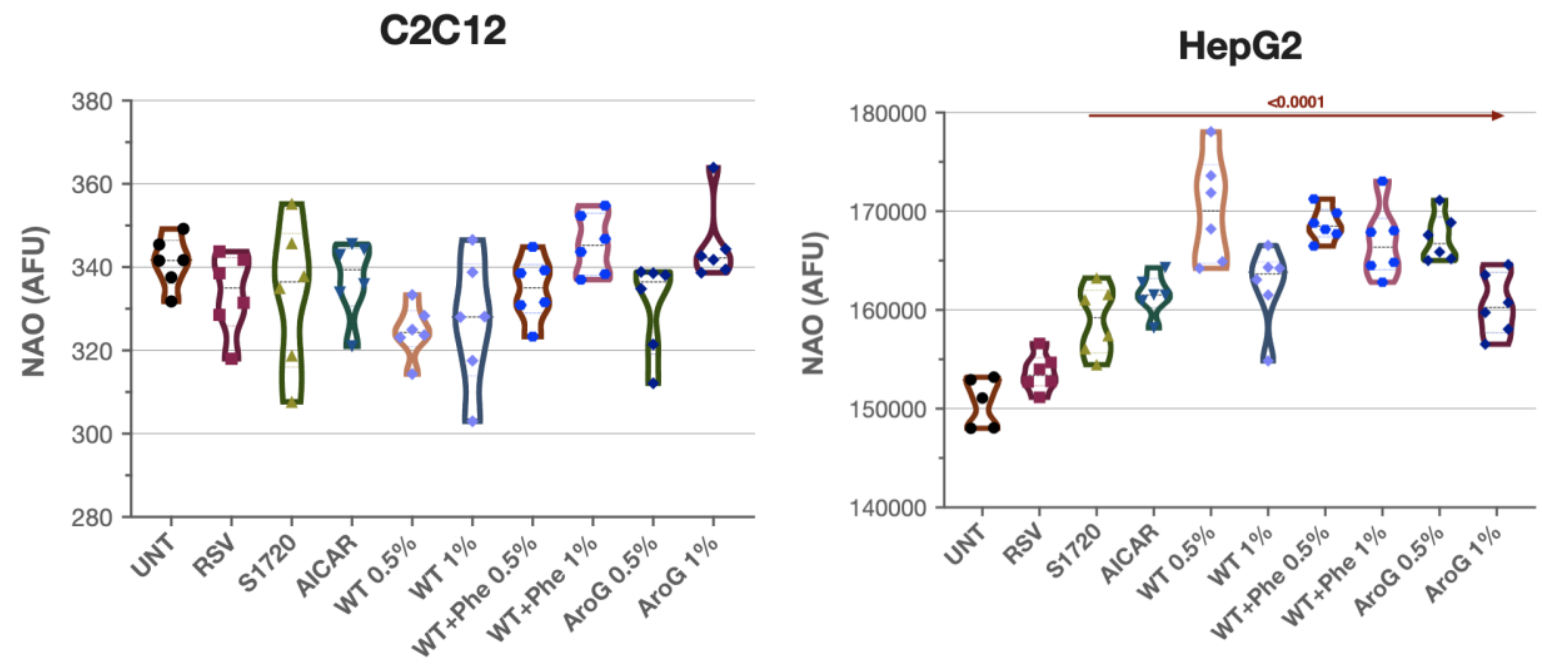

Fig. 4. Mitochondrial mass in cells incubated with GCEs. In this assay, myoblasts (left panel) or hepatocytes (right panel) $\mathrm{NAO}(25 \mathrm{nM})$ was incubated for $30 \mathrm{~min}$ at $37^{\circ} \mathrm{C}$ for $30 \mathrm{~min}$ and washed twice before fluorescence quantification in a plate reader (Ex/Em 495/519 nm). Violin plots depict the average of 25 fields per well per biological replicate with $95 \% \mathrm{Cl}$ at their ends. P-values $(\leq 0.05)$ depicted in red above the data points, were calculated by a one-way ANOVA with Dunnett correction for multiple comparisons using untreated cells (UNT) as control $(n=5-6)$.

Our results indicate that GCEs at $0.5 \%$ or $1 \%$ stimulate mitochondrial biogenesis, particularly in hepatocytes but not myoblasts, suggesting that these cells are more sensitive to PPs (Figure 4). This effect was significantly higher than resveratrol alone or AICAR (5Aminoimidazole-4-carboxamide ribonucleotide), an agonist of AMPK, an enzyme key in sensing the ADP/ATP ratio that usually is activated under conditions of caloric restriction, promoting mitochondrial biogenesis among other changes.

\section{Mitochondrial Respiration}

If the GCEs would increase mitochondrial mass, it is possible that it would affect the rate of oxygen consumption in live cells. To determine this, we used a Seahorse instrument, which estimates the amount of oxygen dissolved in the media within a transient microchamber of just $200 \mu \mathrm{m}$ above the cells, using fluorescent sensor probes sensitive to molecular oxygen dissolved in the media.

This instrument renders valuable information about the mitochondrial respiration in real time. In combination with specific inhibitors of the respiratory chain complex (RCC), it can reveal variables like the basal respiration, the mitochondrial ATP production, the spare respiratory capacity, etc., which in turn can indicate at what step of the oxidative phosphorylation or OXPHOS is changing inside the mitochondria. 
The assay we used is called mito-stress and it relies on the use of four selective inhibitors of the OXPHOS: i) Oligomycin which inhibits the ATP synthase, allowing to estimate the respiration linked to mitochondrial ATP production; ii) BAM15 (N5,N6-bis(2fluorophenyl) $[1,2,5]$ oxadiazolo[3,4-b]pyrazine-5,6-diamine $)^{23}$, an ionophore that specifically dissipates the proton electrochemical potential of the inner mitochondrial membrane, provoking an increase in electron transfer and $\mathrm{O}_{2}$ consumption -which reveals the spare respiratory capacity of the mitochondria-; and iii) Rotenone + Antimycin A, both of which inhibit complexes I and III, respectively, where NADH oxidation and electron transfer are used to pump protons into the intermembrane space of the mitochondria, eventually turning off completely all respiration by the electron transfer chain (ETC). See Figure 5 .

A

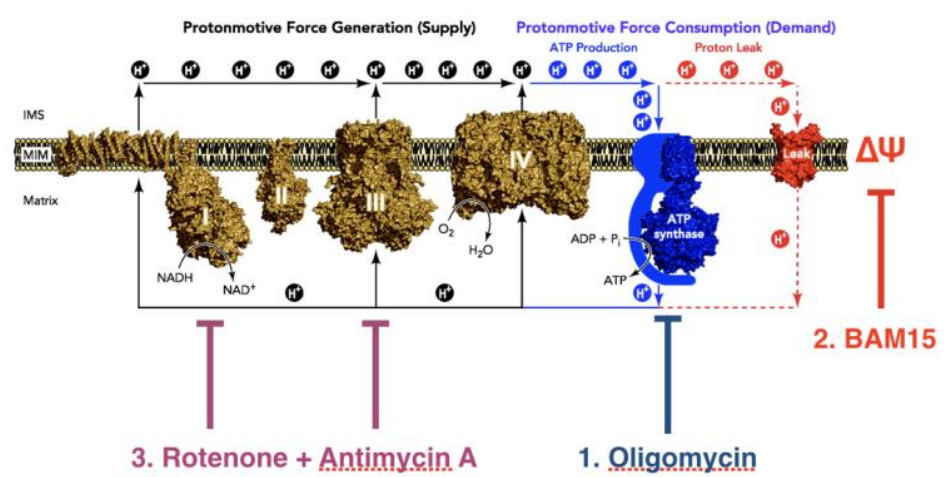

B

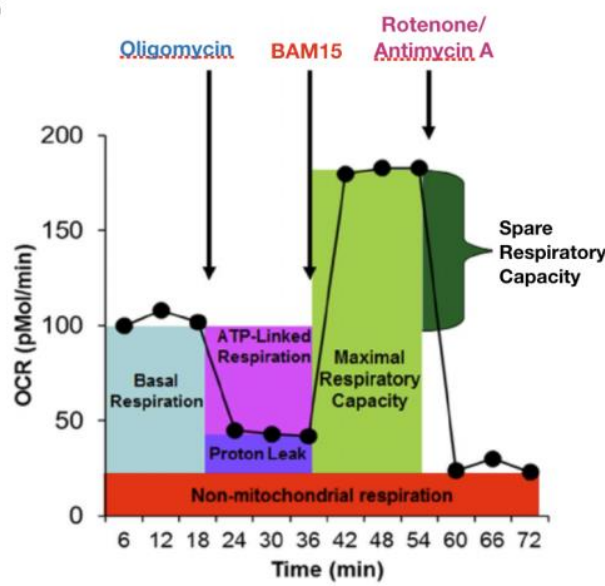

Fig. 5. Principles of the Mito-Stress Test to Determine Mitochondrial Respiratory Capacity as Measured by the Seahorse Platform. A. Schematics of the protein complexes (I-V) involved in the mitochondrial respiratory chain and their inhibitors. B. Oxygen Consumption Rate ( $y$-axis) as determined by the Seahorse platform at various times ( $x$-axis), upon the addition of the inhibitors. The relevant parameters that can be computed from these data are colored.

\section{Glossary}

Oxygen Consumption Rate (OCR). The rate of decrease of oxygen concentration in the assay medium. OCR is a measure of the rate of mitochondrial respiration of the cells.

Extracellular Acidification Rate (ECAR). The rate of increase in proton concentration [or decrease in pH] in the assay medium. OCR is a measure of the rate of glycolysis of the cells.

Group Collection. The collection of individual replicate groups from multiple Excel assigned to a single group. Each Group Collection must have at least 2 assay groups assigned to it from different Excel files.

Basal Respiration. Oxygen consumption used to meet cellular ATP demand and resulting from mitochondrial proton leak. Shows energetic demand of the cell under baseline conditions.

ATP Production. The decrease in oxygen consumption rate upon injection of the ATP synthase inhibitor oligomycin represents the portion of basal respiration that was being used to drive ATP production. Shows ATP produced by the mitochondria that contributes to meeting the energetic needs of the cell.

$\mathrm{H}+$ (Proton) Leak. Remaining basal respiration not coupled to ATP production. Proton leak can be a sign of mitochondrial damage or can be used as a mechanism to regulate the mitochondrial ATP production.

Maximal Respiration. The maximal oxygen consumption rate attained by adding the uncoupler FCCP. FCCP mimics a physiological "energy demand" by stimulating the respiratory chain to operate at maximum capacity, which causes rapid oxidation of substrates (sugars, fats, amino acids) to meet this metabolic challenge. Shows the maximum rate of respiration that the cell can achieve. 
Spare Respiratory Capacity. This measurement indicates the capability of the cell to respond to an energetic demand as well as how closely the cell is to respire to its theoretical maximum. The cell's ability to respond to demand can be an indicator of cell fitness or flexibility.

Non-mitochondrial Oxygen Consumption. Oxygen consumption that persists due to a subset of cellular enzymes that continue to consume oxygen after rotenone and antimycin-A addition. This is important for getting an accurate measure of mitochondrial respiration.

Our results indicate that cells treated with $5 \%$ GCEs recurrently showed lower basal oxygen consumption compared to untreated cells or resveratrol only (Figure 6 ). This effect seemed consistent in both cell lines, but the control in HepG2s yielded a very low signal that the experiment needs to be repeated.
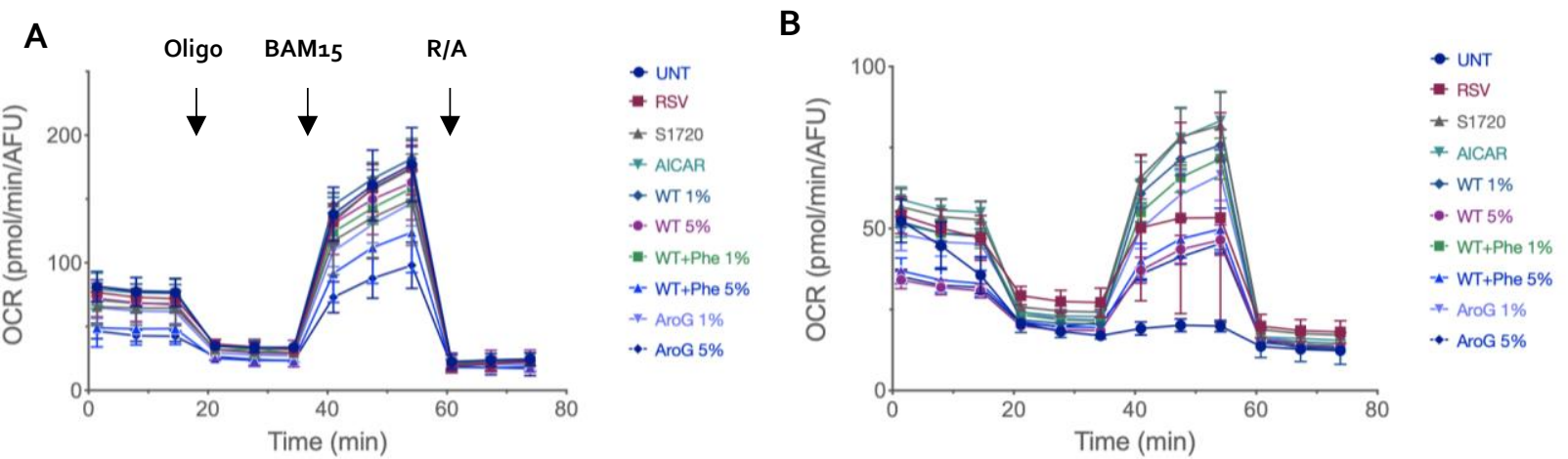

Fig. 6. Seahorse Assay to Determine the Oxygen Consumption Rate (OCR) of Myoblasts and Hepatocytes Incubated with GCEs. Approximately 20,000 ( $\mathrm{C}_{2} \mathrm{C}_{12}$, A) or 40,000 ( $\left.\mathrm{HepG}_{2}, \mathrm{~B}\right)$ cells were incubated in a clear bottom 96 -well plate with $1 \%$ or $5 \%$ GCEs for $24 \mathrm{~h}$ prior to the assay. One hour before the assay, the cells were incubated in a serum-free unbuffered media containing $5.5 \mathrm{mM}$ glucose, $1 \mathrm{mM}$ pyruvate, and $2 \mathrm{mM}$ glutamine, at $37^{\circ} \mathrm{C}$ in the absence of $\mathrm{CO}_{2}$ to avoid acidification of the media by gas exchange. The OCR data was collected every 3 min at $37^{\circ} \mathrm{C}$ in an XFeg 6 Seahorse instrument. Every 9 min, a selective inhibitor of the electron transport chain (ETC) (oligomycin, BAM15, or a rotenone/antimycin A mix) was sequentially injected into every well (indicated by the arrows). At the end of the assay, cell nuclei were stained with CyQuant dye to be able to normalize data by cell number, quantifying fluorescence in a plate reader (Ex/Em 622/645 $\mathrm{nm}$ ). Every point in the plot represents the average of five to six replicates per condition. AFU, arbitrary fluorescence units. Oligo, Oligomycin (1 UM); BAM15, N5,N6-bis(2-Fluorophenyl)[1,2,5]oxadiazolo[3,4-b]pyrazine-5,6-diamine (5 uM); Rot/Ant, Rotenone/Antimycin A (1 UM each). RSV, Resveratrol (12.5 mM), S1720, SIRT1720, (2 UM); AICAR, 5-aminoimidazole-4carboxamide ribonucleotide (50 UM).

A closer look into different parameters of the kinetics of the OCR in myoblasts showed that the extracts derived from WT+Phe and AroG lines at 5\%, significantly lowered basal respiration, proton leak, maximal respiration, and spare respiratory capacity (Fig. 7).

\begin{tabular}{|ll|}
\hline $\begin{array}{l}\text { Parameter Value } \\
\text { Non-mitochondrial oxygen } \\
\text { consumption }\end{array}$ & Equation \\
\hline Basal respiration & $\begin{array}{l}\text { (Last rate measurement before } 1^{\text {st }} \text { injection) - (non-mitochondrial respiration } \\
\text { rate }\end{array}$ \\
\hline Maximal respiration & $\begin{array}{l}\text { (Maximum rate measurement after BAM15 injection) - (Non mitochondrial } \\
\text { respiration) }\end{array}$ \\
\hline $\mathbf{H}^{+}$(proton) leak & $\begin{array}{l}\text { (Minimum rate measurement after Oligomycin injection) - (non- } \\
\text { mitochondrial respiration) }\end{array}$ \\
\hline $\mathbf{A T P}^{\text {(Last rate measurement before Oligomycin injection) - (minimum rate }}$ \\
\hline Spare respiratory capacity & $\begin{array}{l}\text { measurement after Oligomycin injection) } \\
\text { (Maximal respiration) - (Basal respiration) }\end{array}$ \\
\hline Spare respiratory capacity as a \% & (Maximal respiration) / (Basal respiration) $\times 100$ \\
\hline
\end{tabular}




\begin{tabular}{|ll|}
\hline Acute response & $\begin{array}{l}\text { (Last rate measurement before Oligomycin injection) - (last rate } \\
\text { measurement before acute injection }\end{array}$ \\
\hline Coupling efficiency & (ATP Production rate) / (Basal respiration rate) \\
\hline
\end{tabular}

Table 2. Parameters and Equations Used from a Mito-Stress Assay to Profile Mitochondrial Performance. See text for details.

A

Basal Respiration

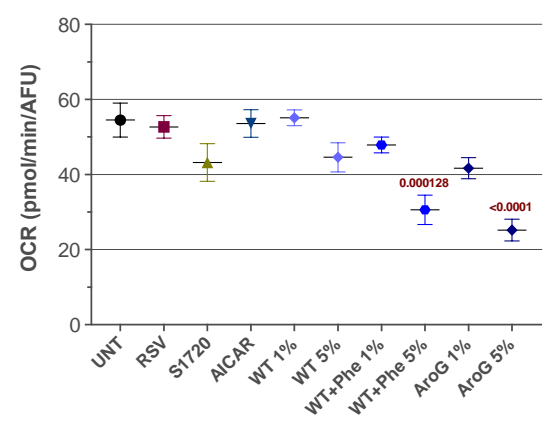

C

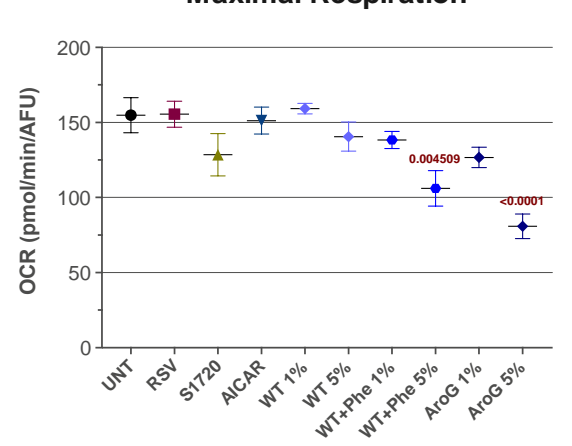

E Non Mitochondrial Oxygen Comsumption

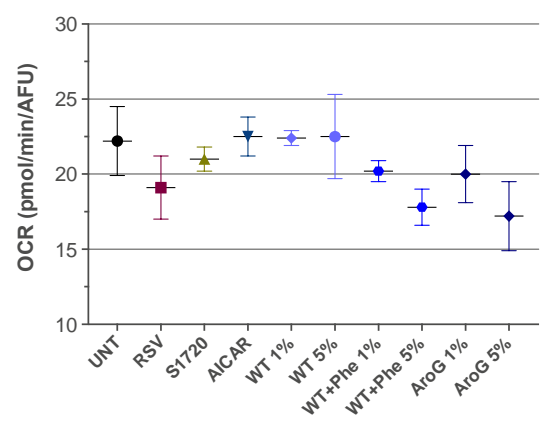

G

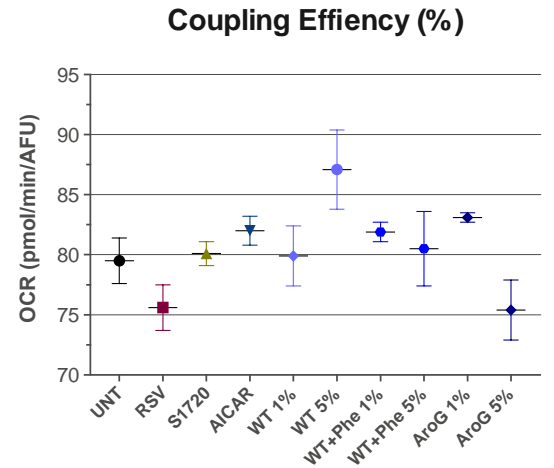

B

Proton Leak

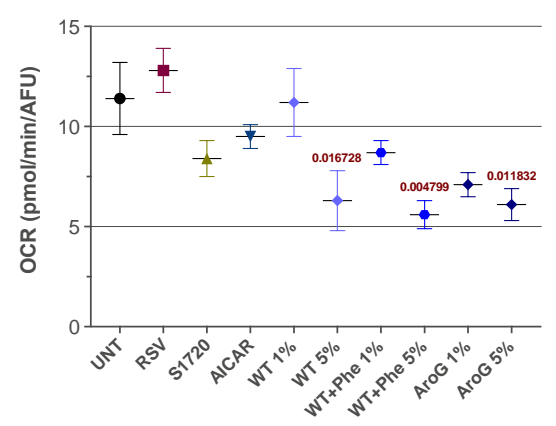

D Spare Respiratory Capacity

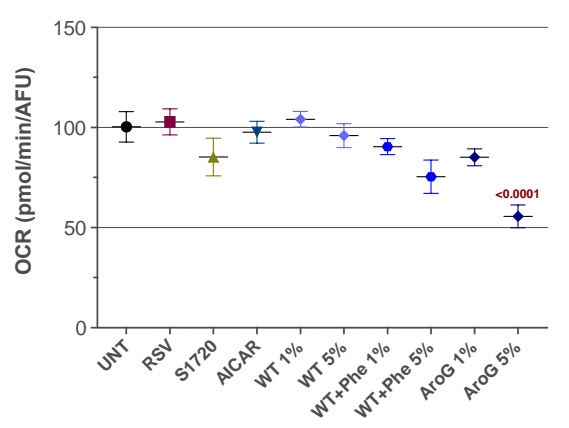

$\mathbf{F}$ ATP Production

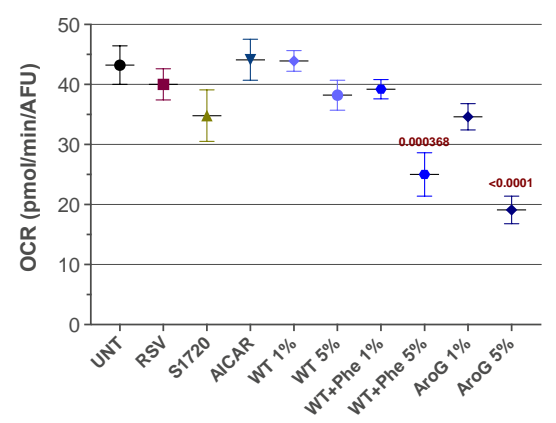

H

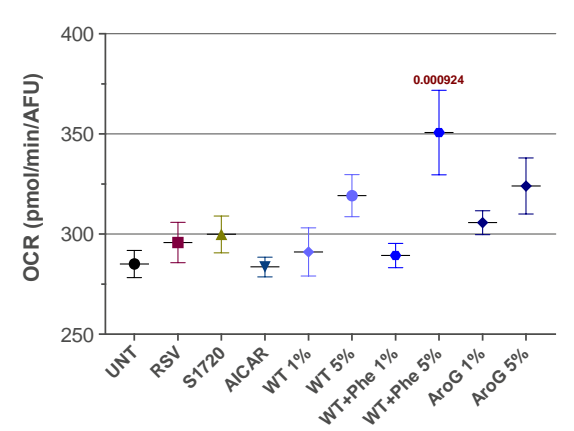

Fig. 7. Mitochondrial Performance in Myoblasts Incubated with GCEs as Determined by the Mito-Stress Assay. Data for every variable was calculated according to the equations on Table 2 using the OCR measured at various time points. P- 
values ( $\leq 0.05$ ) depicted in red above the data points, were calculated by a one-way ANOVA with Dunnett correction for multiple comparisons using untreated cells (UNT) as control $(n=5-6)$.

\section{Mitochondrial Membrane Potential ( $\Psi \mathrm{m})$}

The reduction of maximum respiratory capacity observed could be due to a decrease in the concentration of protons present in the intermembrane space, required to propel the ATP synthase. We then measured the mitochondrial membrane potential using tetramethylrhodamine methyl ester perchlorate (TMRM), a dye that accumulates in this space and whose fluorescence emission linearly correlates with the proton concentration $\underline{24}$.

We found that in the presence of the GCEs, the membrane potential decreased, particularly when used at $5 \%$ of all extracts suggesting that these compounds promote a general reduction of the mitochondrial function, that is accompanied by an increase in mitochondrial mass, possibly as a compensatory mechanism (Fig. 8).

A
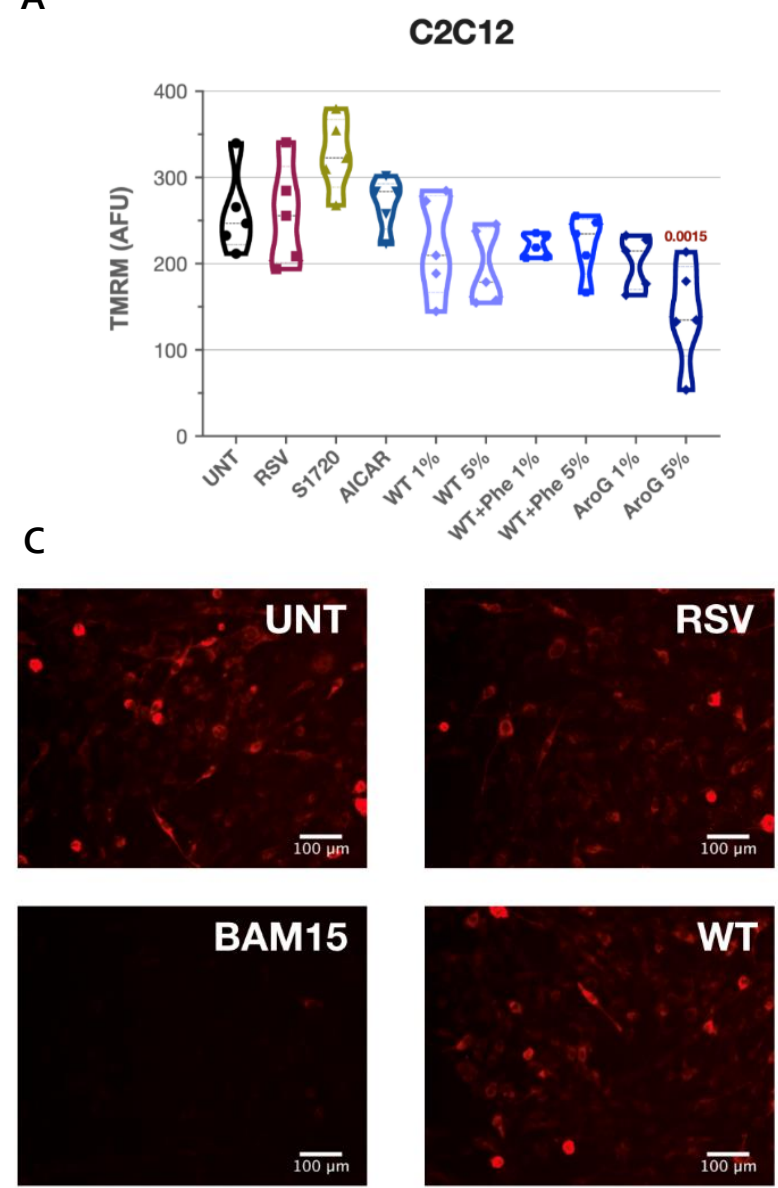
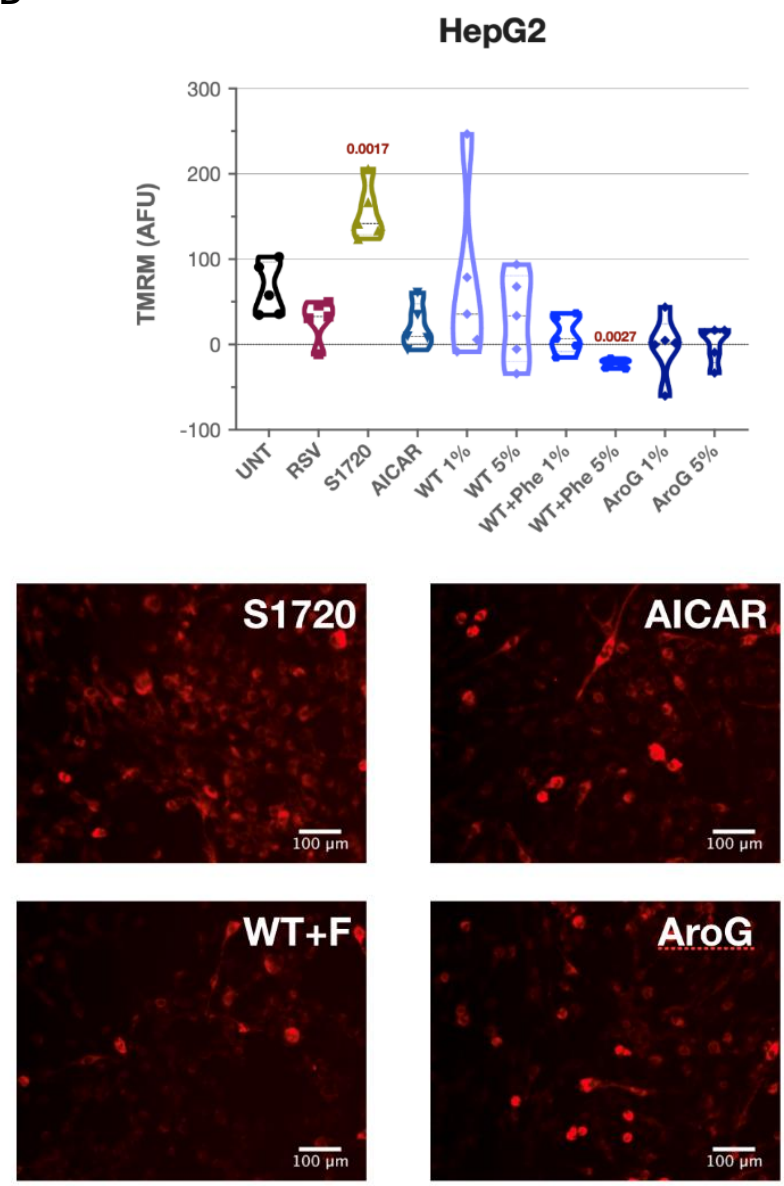

Fig. 8. Mitochondrial Membrane Potential as Determined by TMRM staining. A. Myoblasts or B. Hepatocytes were incubated with $1 \%$ or $5 \%$ of GCEs for $24 \mathrm{~h}$ and stained with $100 \mathrm{nM}$ TMRM for $45 \mathrm{~min}$ ( $\mathrm{n}=6$ per condition). Media was washed twice with phenol red-free media, and fluorescence was measured in a plate reader (Ex/Em 548/574). Violin plots depict the average of 25 fields per well per biological replicate with $95 \% \mathrm{Cl}$ at their ends. P-values ( $\leq 0.05$ ) depicted in red above the data points, were calculated by a one-way ANOVA with Dunnett correction for multiple comparisons using untreated cells (UNT) as control $(n=5-6)$. C. Representative images of the cells were taken in an inverted epifluorescence microscope with a $20 X$ objective using a FITC filter. BAM15 $(5 \mathrm{UM})$ was used as a negative control and was incubated for 10 min before the assay on separate wells.

Scavenging free oxygen radicals 
It has been reported that polyphenolic compounds from grape display an anti-oxidant activity $3,16,25,26$. This property is useful in conditions when the production of free radicals like hydroxyl $\left(\mathrm{HO}^{*}\right)$, superanion oxide $\left(\mathrm{O}_{2}{ }^{--}\right)$, or ferryl ion $\left(\mathrm{FeO}^{2+}\right)$ are enhanced 27 , like in aged tissues where poorly regulated mitochondria transfers an excess of electrons to molecular oxygen in Complex I producing superoxide, or due to the dysregulation of free radical scavenging enzymes like catalase, glutathione peroxidases and peroxiredoxins. These radicals rapidly oxidize fatty acids at the cell and organelle membranes, ultimately damaging genomic and mitochondrial DNA.

To test the antioxidant capacity of the GCEs in culture, we used a molecular sensor (CellROX Green) that becomes fluorescent only with subsequent binding to DNA, limiting its presence to the nucleus or mitochondria upon oxidation.

A
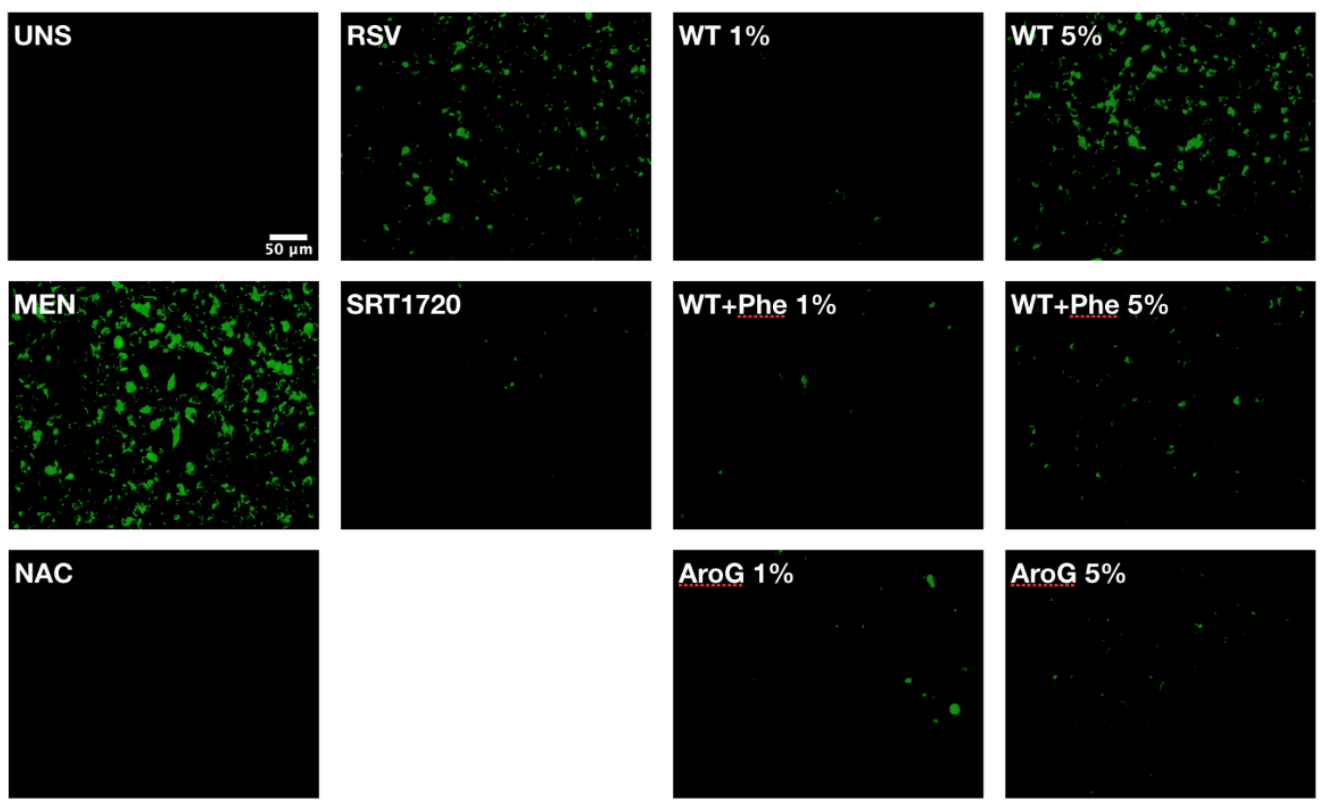

B

HepG2

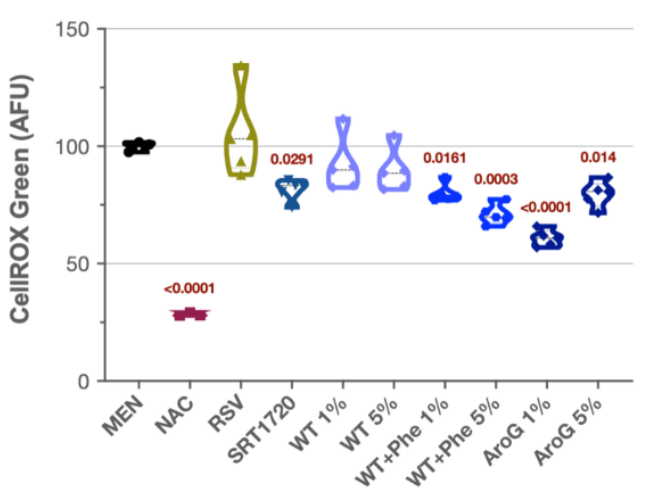

Fig. 9. Anti-oxidant Capacity of Hepatocytes Cells Incubated with GCEs. HepG2 cells were plated in a clear-bottom 96 well plate and incubated with $1 \%$ or $5 \%$ of GCEs for $24 \mathrm{~h}$. Media was washed and replaced with the oxidant menadione (50 UM) for 1 h, followed by a 30 min staining with CellROX Green for 30 min. Fluorescence was measured in a plate reader (Ex/Em 485/520). A. Images of the cells were taken in an inverted epifluorescence microscope with a $20 X$ objective using a FITC filter. B. Violin plots depict the average of 25 fields per well per biological replicate with $95 \% \mathrm{Cl}$ at their ends. P-values $(\leq 0.05)$ depicted in red above the data points, were calculated by a one-way ANOVA with Dunnett correction for multiple comparisons using menadione-treated cells (MEN) as control $(n=5-6)$. The anti-oxidant $N$-acetyl cysteine (NAC) was used as a negative control. RSV was used at $12.5 \mathrm{UM}$; SRT1720 was used at 2 UM. 
In order to increase the production of free radicals in the cells, we used menadione, a polycyclic aromatic ketone that can function as a precursor in the synthesis of Vitamin K. At high doses this compound generates intracellular ROS at multiple cellular sites through futile redox cycling, mainly producing hydrogen peroxide and ferryl ion through the Fenton reaction $\underline{28}, \underline{29}$.

Our findings indicate that the GCEs confer protection against menadione when incubated for $24 \mathrm{~h}$ in hepatocytes, even after the media has been replaced. We did not use $\mathrm{C}_{2} \mathrm{C}_{12}$ cells since they were too sensitive to menadione treatment.

Interestingly, both the WT+Phe and AroG extracts displayed higher anti-oxidant activity, though not at the same dilutions. The AroG line showed a larger decrease in CellROX Green staining at $1 \%$ but not at $5 \%$. This could be due to the fact that cells treated with $5 \% \mathrm{GCE}$ were less viable and with the possibility that their damaged mitochondria would have enhanced the oxidative effect of menadione through other mechanisms (e.g. by a reduction of the Cytochrome $\mathrm{C}$ which also helps in limiting free radicals in the mitochondria or by the reduction of other anti-oxidant enzymes).

\section{Summary}

-Grape cells extracts are deleterious in rapidly dividing cells, in a dose-dependent manner. -These affect cellular viability and greatly reduce mitochondrial activity, particularly in hepatocarcinoma cells.

-The potency of the toxic effect of both WT+Phe and AroG lines is higher than WT alone. -These same extracts protect cells against potent oxidants like menadione. 


\section{References}

1

Milne, J. C. et al. Small molecule activators of SIRT1 as therapeutics for the treatment of type 2 diabetes. Nature 450, 712-716, doi:10.1038/natureo6261 (2007).

Minor, R. K. et al. SRT1720 improves survival and healthspan of obese mice. Sci Rep 1, 70, doi:10.1038/srepooo70 (2011).

Xia, E. Q., Deng, G. F., Guo, Y. J. \& Li, H. B. Biological activities of polyphenols from grapes. Int J Mol Sci 11, 622-646, doi:10.339o/ijms11020622 (2010).

Singh, C. K., Siddiqui, I. A., El-Abd, S., Mukhtar, H. \& Ahmad, N. Combination chemoprevention with grape antioxidants. Mol Nutr Food Res 60, 1406-1415, doi:10.1002/mnfr.201500945 (2016).

Paller, C. J. et al. A phase I study of muscadine grape skin extract in men with biochemically recurrent prostate cancer: Safety, tolerability, and dose determination. Prostate 75, 1518-1525, doi:10.1002/pros.23024 (2015).

Olaku, O. O., Ojukwu, M. O., Zia, F. Z. \& White, J. D. The Role of Grape Seed Extract in the Treatment of Chemo/Radiotherapy Induced Toxicity: A Systematic Review of Preclinical Studies. Nutr Cancer 67, 730-740, doi:10.1080/01635581.2015.1029639 (2015).

Anhe, F. F. et al. Gut Microbiota Dysbiosis in Obesity-Linked Metabolic Diseases and Prebiotic Potential of Polyphenol-Rich Extracts. Curr Obes Rep 4, 389-400, doi:10.1007/s13679-015-0172-9 (2015).

Madrigal-Santillan, E. et al. Review of natural products with hepatoprotective effects. World J Gastroenterol 20, 14787-14804, doi:10.3748/wjg.v20.i40.14787 (2014).

Marchi, P., Paiotti, A. P., Artigiani Neto, R., Oshima, C. T. \& Ribeiro, D. A. Concentrated grape juice (G8000) reduces immunoexpression of iNOS, TNF-alpha, COX-2 and DNA damage on 2,4,6-trinitrobenzene sulfonic acid-induced-colitis. Environ Toxicol Pharmacol 37, 819-827, doi:10.1016/j.etap.2014.02.006 (2014).

Asseburg, H. et al. Effects of Grape Skin Extract on Age-Related Mitochondrial Dysfunction, Memory and Life Span in C57BL/6J Mice. Neuromolecular Med 18, 378395, doi:10.1007/s12017-016-8428-4 (2016).

1 Ferhi, S. et al. Total Phenols from Grape Leaves Counteract Cell Proliferation and Modulate Apoptosis-Related Gene Expression in MCF-7 and HepG 2 Human Cancer Cell Lines. Molecules 24, doi:10.3390/molecules24030612 (2019).

de Sales, N. F. F. et al. Anthocyanin-Rich Grape Pomace Extract (Vitis vinifera L.) from Wine Industry Affects Mitochondrial Bioenergetics and Glucose Metabolism in Human Hepatocarcinoma HepG2 Cells. Molecules 23, doi:10.339o/molecules23030611 (2018). 
13 Bak, M. J. et al. Antioxidant and Hepatoprotective Effects of Procyanidins from Wild Grape (Vitis amurensis) Seeds in Ethanol-Induced Cells and Rats. Int J Mol Sci 17, doi:10.339o/ijms17050758 (2016).

14 Goutzourelas, N. et al. Effects of polyphenolic grape extract on the oxidative status of muscle and endothelial cells. Hum Exp Toxicol 33, 1099-1112, doi:10.1177/og60327114533575 (2014).

15 Camont, L. et al. Simple spectrophotometric assessment of the trans-/cis-resveratrol ratio in aqueous solutions. Anal Chim Acta 634, 121-128, doi:10.1016/j.aca.2008.12.003 (2009).

16 Biais, B. et al. Antioxidant and Cytoprotective Activities of Grapevine Stilbenes. J Agric Food Chem 65, 4952-4960, doi:10.1021/acs.jafc.7bo1254 (2017).

17 Britton, R. G., Kovoor, C. \& Brown, K. Direct molecular targets of resveratrol: identifying key interactions to unlock complex mechanisms. Ann N Y Acad Sci 1348, 124-133, doi:10.1111/nyas.12796 (2015).

18 Bao, L. et al. Grape seed proanthocyanidin extracts ameliorate podocyte injury by activating peroxisome proliferator-activated receptor-gamma coactivator 1alpha in low-dose streptozotocin-and high-carbohydrate/high-fat diet-induced diabetic rats. Food Funct 5, 1872-1880, doi:10.1039/c4fooo340c (2014).

19 Liu, Q. et al. Grape seed extract ameliorates bleomycin-induced mouse pulmonary fibrosis. Toxicol Lett 273, 1-9, doi:10.1016/j.toxlet.2017.03.012 (2017).

20 Del Pino-Garcia, R. et al. Chemopreventive Potential of Powdered Red Wine Pomace Seasonings against Colorectal Cancer in HT-29 Cells. J Agric Food Chem 65, 66-73, doi:10.1021/acs.jafc.6b04561 (2017).

21 Stagos, D. et al. Antiangiogenic potential of grape stem extract through inhibition of vascular endothelial growth factor expression. J Physiol Pharmacol 65, 843-852 (2014).

22 Sahpazidou, D. et al. Anticarcinogenic activity of polyphenolic extracts from grape stems against breast, colon, renal and thyroid cancer cells. Toxicol Lett 230, 218-224, doi:10.1016/j.toxlet.2014.01.042(2014).

23 Kenwood, B. M. et al. Identification of a novel mitochondrial uncoupler that does not depolarize the plasma membrane. Mol Metab 3, 114-123, doi:10.1016/j.molmet.2013.11.005 (2014).

24 Cottet-Rousselle, C., Ronot, X., Leverve, X. \& Mayol, J. F. Cytometric assessment of mitochondria using fluorescent probes. Cytometry $A$ 79, 405-425, doi:10.1002/cyto.a.21061 (2011). 
25 Ferri, M. et al. White grape pomace extracts, obtained by a sequential enzymatic plus ethanol-based extraction, exert antioxidant, anti-tyrosinase and antiinflammatory activities. N Biotechnol 39, 51-58, doi:10.1016/j.nbt.2017.07.002 (2017).

26 Razmaraii, N., Babaei, H., Mohajjel Nayebi, A., Ahdi Khosroshahi, S. \& Farajnia, S. Evaluation of Cytotoxic Effect and Antioxidant Activity of Grape Seed Extract, Crocin, and Phenytoin. Arch Razi Inst 72, 181-187, doi:10.22092/ari.2017.111609 (2017).

27 Koppenol, W. H. \& Hider, R. H. Iron and redox cycling. Do's and don'ts. Free Radic Biol Med 133, 3-10, doi:10.1016/j.freeradbiomed.2018.09.022 (2019).

28 Loor, G. et al. Menadione triggers cell death through ROS-dependent mechanisms involving PARP activation without requiring apoptosis. Free Radic Biol Med 49, 19251936, doi:10.1016/j.freeradbiomed.2010.09.021 (2010).

29 Criddle, D. N. et al. Menadione-induced reactive oxygen species generation via redox cycling promotes apoptosis of murine pancreatic acinar cells. J Biol Chem 281, 40485-40492, doi:10.1074/jbc.M607704200 (2006). 